\title{
Rapid Detection of Tetracycline Residues in Duck Meat Using Surface Enhanced Raman Spectroscopy
}

\author{
Jinhui Zhao, ${ }^{1}$ Ping Liu, ${ }^{2}$ Haichao Yuan, ${ }^{1}$ Yijie Peng, ${ }^{1}$ Qian Hong, ${ }^{1}$ and Muhua Liu ${ }^{1}$ \\ ${ }^{1}$ Optics-Electrics Application of Biomaterials Lab, College of Engineering, Jiangxi Agricultural University, Nanchang 330045, China \\ ${ }^{2}$ College of Animal Science and Technology, Jiangxi Agricultural University, Nanchang 330045, China \\ Correspondence should be addressed to Muhua Liu; suikelmh@sohu.com
}

Received 25 August 2016; Accepted 9 October 2016

Academic Editor: Christoph Krafft

Copyright (c) 2016 Jinhui Zhao et al. This is an open access article distributed under the Creative Commons Attribution License, which permits unrestricted use, distribution, and reproduction in any medium, provided the original work is properly cited.

\begin{abstract}
A rapid detection method based on surface enhanced Raman spectroscopy (SERS) was proposed in this paper in order to realize the detection of tetracycline residues in duck meat. Firstly, surface enhanced Raman spectra characteristics of tetracycline aqueous solution, duck meat extract, and duck meat extract containing tetracycline were analyzed. Secondly, the effect of the addition amount of duck meat extract containing tetracycline on SERS intensity and the effect of the adsorption time on SERS intensity were discussed, respectively. Thirdly, SERS intensity ratio at 1272 and $1558 \mathrm{~cm}^{-1}\left(I_{1272} / I_{1558}\right)$ was used to establish the SERS calibration curve. A good linearity relationship between the tetracycline concentration in duck meat extract and $I_{1272} / I_{1558}$ was obtained, and the linear regression equation and the correlation coefficient $(r)$ were $y=0.0177 x+0.1213$ and 0.950 , respectively. The average recovery of tetracycline in duck meat extract was 101 108\% with relative standard deviation (RSD) of 2.4 4.6\%. The experimental results showed that the method proposed in this paper was a good detection scheme for the rapid detection of tetracycline residues in duck meat.
\end{abstract}

\section{Introduction}

Tetracycline $\left(\mathrm{C}_{22} \mathrm{H}_{24} \mathrm{~N}_{2} \mathrm{O}_{8}\right)$, which is a kind of broadspectrum tetracycline antibiotics, is often used to prevent and treat the duck diseases or is added to the duck feedstuff as the additive. However, tetracycline residues content in duck meat easily exceeds the standard within legal limits due to the unsuitable usage of tetracycline, and the excessive tetracycline residues in duck meat may further cause some potential harm to human health, such as kidney toxicity and fetal malformation $[1,2]$. Therefore, the regulations for the Maximum Residue Limits (MRL) in meat have been established in China, the EU, and the USA $[1,3]$. For instance, tetracycline residues content in duck meat in China and USA cannot exceed 0.1 and $0.2 \mathrm{ppm}$, respectively $[1,3]$.

It is highly imperative to detect all the duck products in advance in order to prevent the products containing the tetracycline residues from entering the markets. The conventional detection methods for the antibiotic residues in meat include enzyme-like immunosorbent assay [4-6], microbiological method $[7,8]$, and physical and chemical detection method [9]. Although these methods have high detection precision, they cannot meet the rapid, mass, and field detection requirements of tetracycline residues in duck meat owing to the tedious pretreatment process or the timeconsuming detection process and so forth. Hence, it is highly needed to investigate a rapid detection method of tetracycline residues in duck meat.

Surface enhanced Raman spectroscopy (SERS) is a highly specific and sensitive method for the detection of chemical and biochemical compounds in chemistry, microbiology, pharmacology, biochemistry, and environmental science $[10,11]$. Zhang et al. utilized SERS to analyze prohibited aquaculture drugs including enrofloxacin, furazolidone, and malachite green in fish products [11]. Li et al. applied SERS to detect the residuals of the prohibited and restricted drugs including malachite green and crystal violet in fish muscle, and the lowest concentrations detected were 1.0 and $20 \mu \mathrm{g} / \mathrm{kg}$, respectively [12]. Ma et al. used SERS to detect sulfamerazine and sulfamethazine in fish, and the detection limits were 0.16 and $0.59 \mathrm{ppm}$, respectively [13]. Li et al. applied improved surface enhanced Raman scattering on microscale Au hollow 
spheres to detect different concentrations of tetracycline aqueous solution, and a good linear response was obtained [14]. Compared to the SERS detection of tetracycline aqueous solution, the SERS detection condition of tetracycline residues in duck meat is more complicated. For instance, the composition of duck meat can have effect on intensities of tetracycline SERS characteristic peaks; even some tetracycline SERS characteristic peaks disappear. Moreover, some tetracycline SERS characteristic peaks can overlap with SERS peaks of the composition of duck meat. So far, the relevant literature about the detection of tetracycline residues in duck meat using SERS was not reported.

The objective of this paper was to investigate the rapid detection method of tetracycline residues in duck meat using SERS. Firstly, SERS spectra characteristics of tetracycline aqueous solution, duck meat extract, and duck meat extract containing tetracycline were analyzed. Secondly, the effect of the addition amount of duck meat extract containing tetracycline on SERS intensity (peak height) and the effect of the adsorption time on SERS intensity were explored, respectively. Lastly, the linear regression equation between the tetracycline concentration in duck meat extract and SERS intensity ratio at 1272 and $1558 \mathrm{~cm}^{-1}\left(I_{1272} / I_{1558}\right)$ was established to predict the tetracycline residues in duck meat.

\section{Materials and Methods}

2.1. Materials and Reagents. Sheldrakes were purchased from the vegetable market of Jiangxi Agricultural University. Tetracycline $(98.0 \%)$ was purchased from Standard Substances Network of China. OTR202 (gold nanoparticles), and OTR103 (gold colloid enhancement reagent) were purchased as SERS enhancement substrate from Opto Trace Technologies, Inc. Ethyl acetate was analytical reagent grade. Ultrapure water was obtained using T10 laboratory water purifier (Hunan Kertone Water Treatment Co., Ltd.) for the preparation of all aqueous solutions. All chemicals are of analytical reagent grade and used without further purification.

2.2. Instruments. RamTracer ${ }^{\circledR}-200$ portable Raman spectrometer (Opto Trace Technologies, Inc.), T6 series UVVis spectrophotometer (Beijing Purkinje General Instrument Co., Ltd.), JK-50B ultrasonic cleaner (Hefei Jinnike Machinery Co., Ltd.), FA1004B electronic weigher (Shanghai Shangping instrument Co., Ltd.), T10 laboratory water purifier, JW1024 low speed centrifuge (Anhui Jiaven Equipment Industry Co., Ltd.), JJ-2B tissue disintegrator (Jintan Jinnan Instrument Factory), and VORTEX-5 whirlpool mixer (Haimen Kylin-Bell Lab Instruments Co., Ltd.) were used in this study.

\subsection{Experimental Methods}

(1) Preparation of duck meat extract: firstly, the breast meats were removed from the sheldrakes and the membranes were eliminated from the breast meats for the following experiment. Secondly, $200 \mathrm{~g}$ of duck breast meat was crushed in meat emulsion by using tissue disintegrator, and then the meat emulsion was cryopreserved below $-18^{\circ} \mathrm{C}$. Thirdly, $5 \mathrm{~g}$ of meat emulsion and $20 \mathrm{~mL}$ of ethyl acetate were added into $50 \mathrm{~mL}$ centrifuge tube every time. Fourthly, the supernatant was taken out after whirlpool mixing for 2 minutes, oscillating for 10 minutes, and centrifuging ( $4500 \mathrm{r} / \mathrm{min}$ ) for 15 minutes. Fifthly, the residues were extracted for two times using the same method, and then two extracted supernatants were mixed together. Lastly, the supernatant was filtered with rapid filter papers.

(2) Preparation of tetracycline standard solution: $5 \mathrm{mg}$ of tetracycline was added into $50 \mathrm{~mL}$ brown volumetric flask, and then $100 \mathrm{mg} / \mathrm{L}$ tetracycline standard solution was obtained after ultrasonic dissolving with ultrapure water. Subsequently, $100 \mathrm{mg} / \mathrm{L}$ tetracycline standard solution was diluted to different concentrations with ultrapure water during the course of the experiment.

(3) Preparation of spiked samples: $5 \mathrm{mg}$ of tetracycline was added into $50 \mathrm{~mL}$ brown volumetric flask, and then the spiked sample containing $100 \mathrm{mg} / \mathrm{L}$ tetracycline was obtained after ultrasonic dissolving with duck meat extract. Subsequently, the spiked sample containing $100 \mathrm{mg} / \mathrm{L}$ tetracycline was diluted to different tetracycline concentrations by using duck meat extract in the following experiment.

(4) $500 \mu \mathrm{L}$ of OTR $202,20 \mu \mathrm{L}$ of the analyzed solution, and $100 \mu \mathrm{L}$ of OTR103 were added to $2 \mathrm{~mL}$ sample bottle in turn, and then SERS spectra were collected after this system was well blended. SERS spectra were collected 5 times for each sample, and their average intensities were used as original SERS spectrum intensities of each sample. Spectral range of 400 $1800 \mathrm{~cm}^{-1}$ was used as the following analysis of SERS spectra.

(5) The parameters of portable Raman spectrometer were set as follows: laser power was $200 \mathrm{~mW}$, laser wavelength was $785 \mathrm{~nm}$, the scanning range of Raman wavelength was $100 \sim 3300 \mathrm{~cm}^{-1}$, spectral resolution was $6 \mathrm{~cm}^{-1}$, the range of Raman intensity was $0 \sim$ 60000 a.u., and the integration time was $10 \mathrm{sec}$.

\section{Results and Discussion}

3.1. Pretreatment of SERS Spectra. The original SERS spectrum of duck meat extract containing $12.0 \mathrm{mg} / \mathrm{L}$ tetracycline was showed in Figure 1(a). As seen from Figure 1(a), the original SERS spectrum included not only Raman signal of the sample detected but also fluorescence and all other kinds of background signals. Thus, it was indispensable to reduce the effect of the fluorescence and all other kinds of background signals on SERS analysis in order to improve the reliability of SERS qualitative, semiquantitative, or quantitative analysis. Adaptive iteratively reweighted penalized least squares (airPLS), which need not any user intervention and initial information, is a highly effective background subtraction method $[15,16]$. So, air-PLS was employed to remove the fluorescence and all other kinds of background signals in 


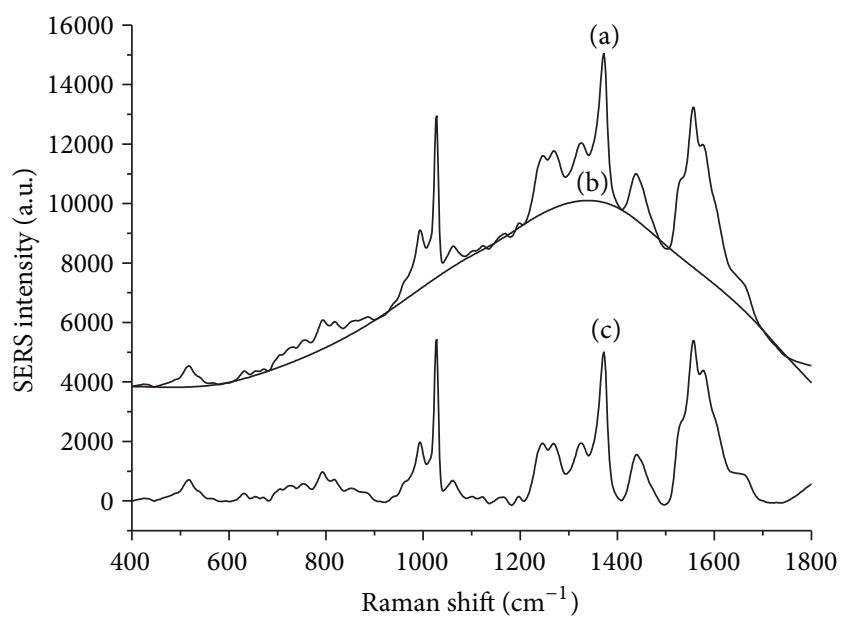

Figure 1: Result of background subtraction using air-PLS. (a) Original SERS spectrum; (b) background signals fitted; (c) SERS spectrum subtracted by background signals.

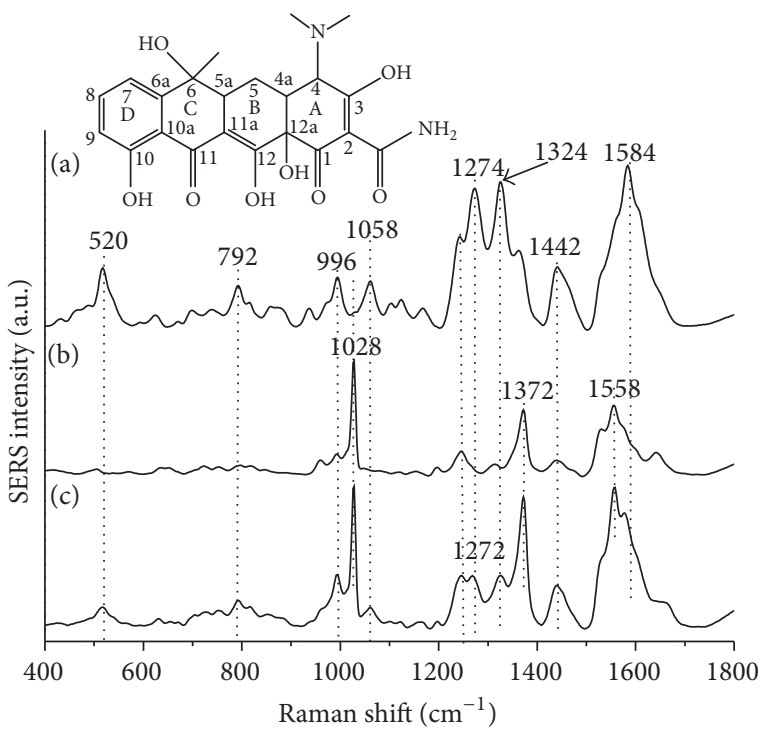

FIGURE 2: SERS spectra of (a) tetracycline aqueous solution, (b) duck meat extract, and (c) duck meat extract containing tetracycline. The inset shows that tetracycline is a derivative of naphthacene according to the chemical structure of tetracycline.

this paper. As showed from Figure 1(c), the fluorescence and all other kinds of background signals on curve (a) were subtracted perfectly by using air-PLS under the condition of keeping the peak shapes of SERS spectra.

\subsection{SERS Spectra Characteristics of Samples. The OTR202} has a strong absorption peak at $544 \mathrm{~nm}$ according to UVVis absorption spectra of OTR202, which is the typical surface plasmon resonance absorption of gold nanoparticles. Moreover, its half-peak width is approximately $79 \mathrm{~nm}$.

SERS spectra of tetracycline aqueous solution, duck meat extract, and duck meat extract containing tetracycline were showed in Figure 2. The major SERS characteristic peaks of tetracycline aqueous solution could be observed at 520, 792,

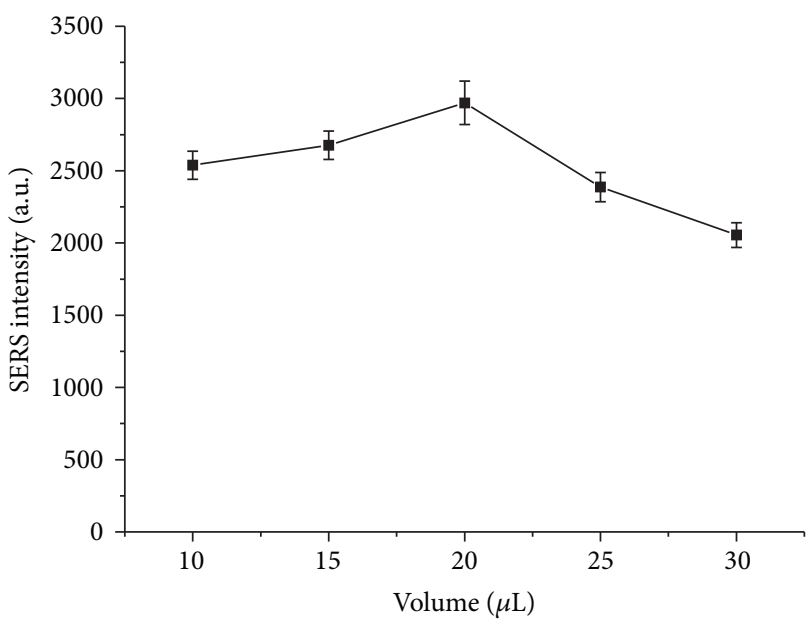

Figure 3: Effect of the addition amount of duck meat extract containing tetracycline on SERS intensity.

996, 1058, 1274, 1324, and $1584 \mathrm{~cm}^{-1}\left( \pm 3 \mathrm{~cm}^{-1}\right)$ in Figure 2(a). SERS characteristic peak of tetracycline aqueous solution at $1274 \mathrm{~cm}^{-1}$ has blue-shifted to $1272 \mathrm{~cm}^{-1}$ on SERS spectrum of duck meat extract containing tetracycline, which was probably caused by the charge transfer that took place between gold nanoparticles and tetracycline [13]. The main SERS characteristic peaks of duck meat extract could be seen at 1028,1372 , and $1558 \mathrm{~cm}^{-1}\left( \pm 3 \mathrm{~cm}^{-1}\right)$ in Figure 2(b). As showed in Figure 2(c), SERS characteristic peaks of tetracycline aqueous solution at 520,1058, and $1274 \mathrm{~cm}^{-1}$, which did not overlap with SERS characteristic peaks of duck meat extract, appeared on SERS spectrum of duck meat extract containing tetracycline. So, SERS characteristic peaks at 520, 1058 , and $1272 \mathrm{~cm}^{-1}$ could be selected as SERS characteristic peaks for the detection of tetracycline residues in duck meat extract. Tetracycline is a derivative of naphthacene according to the chemical structure of tetracycline in Figure 2(a). The characteristic peak at $1058 \mathrm{~cm}^{-1}$ was attributed to stretching vibration of CO3 $[17,18]$. The characteristic peak at $1272 \mathrm{~cm}^{-1}$ was ascribed to bending vibration of $\mathrm{CH} 4,4 \mathrm{a}, 5,5 \mathrm{a}, \mathrm{OH} 12$, and amid-NH and stretching vibration of CO10, $\mathrm{CO} 3, \mathrm{CH} 7,8,9$, amid-NC, $\mathrm{C} 4 \mathrm{aC} 5$, and benzene ring $\mathrm{D}[17,18]$. In conclusion, it was entirely feasible, at least in theory, to detect tetracycline residues in duck meat using SERS according the above analysis.

3.3. Effect of Addition Amount of Samples on SERS Intensity. To analyze the effect of the addition of the samples on SERS intensity, SERS intensity at $1272 \mathrm{~cm}^{-1}$ was investigated under the condition of the different addition amounts $(10,15,20,25$, and $30 \mu \mathrm{L}$ ) of duck meat extract containing tetracycline and the fixed volumes of OTR202 $(500 \mu \mathrm{L})$ and OTR103 $(100 \mu \mathrm{L})$. As showed from Figure 3, SERS intensities at $1272 \mathrm{~cm}^{-1}$ were different when different volumes of duck meat extract containing tetracycline were mixed with gold nanoparticles. The possible reason was that their mixture ratio had the significant impact on adsorption effect and then affected SERS intensity. SERS intensity at $1272 \mathrm{~cm}^{-1}$ was strongest 


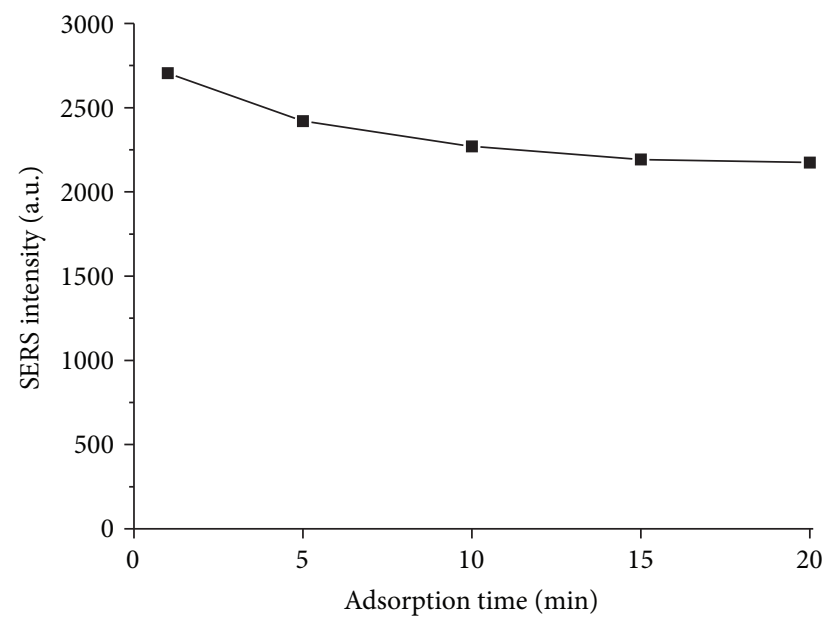

FIGURE 4: Effect of adsorption time on SERS intensity.

while the addition amount of duck meat extract containing tetracycline was $20 \mu \mathrm{L}$. This showed that adsorption effect on the detected sample was best when the volume of duck meat extract containing tetracycline was $20 \mu \mathrm{L}$. Therefore, $20 \mu \mathrm{L}$ was determined as the addition amount of duck meat extract containing tetracycline.

3.4. Effect of Adsorption Time on SERS Intensity. In order to study the effect of adsorption time on SERS intensity, the change of SERS intensity at $1272 \mathrm{~cm}^{-1}$ with adsorption time was explored on the condition of $500 \mu \mathrm{L}$ of OTR202, $20 \mu \mathrm{L}$ of the same concentration of the sample detected, and $100 \mu \mathrm{L}$ of OTR103. With the increases of adsorption time between duck meat extract containing tetracycline and SERS active substrate, SERS intensities of the mixture solution at $1272 \mathrm{~cm}^{-1}$ gradually reduced (Figure 4). This might be because the aggregation was produced to some extent after gold nanoparticles were mixed with duck meat extract containing tetracycline, and the enhancement effects, which were produced by combining active hotspots determining SERS enhancement effects with duck meat extract containing tetracycline, decreased gradually along with the increases of adsorption time. SERS intensity at $1272 \mathrm{~cm}^{-1}$ was strongest when adsorption time was $1 \mathrm{~min}$. This indicated that SERS enhancement effects, which were produced by combining active hotspots with duck meat extract containing tetracycline, were best when adsorption time was $1 \mathrm{~min}$. Therefore, $1 \mathrm{~min}$ was ascertained as the best adsorption time.

3.5. SERS Calibration Curve and Predicted Results. SERS spectra of duck meat extract containing different tetracycline concentrations were showed in Figure 5. SERS characteristic peak of tetracycline aqueous solution at $1272 \mathrm{~cm}^{-1}$ could be observed on SERS spectrum of duck meat extract containing tetracycline $(4 \mathrm{mg} / \mathrm{L})$, and the peak position at $1272 \mathrm{~cm}^{-1}$ was stable. This illustrated that OTR202 and OTR103 could be used as SERS active substrate for the detection of tetracycline residues in duck meat. Thus, $1272 \mathrm{~cm}^{-1}$ could be selected as the characteristic peak of semiquantitative or quantitative analysis of tetracycline residues in duck meat.
TABLE 1: Predicted results of tetracycline in duck meat $(n=5)$.

\begin{tabular}{lccc}
\hline $\begin{array}{l}\text { Spiked } \\
\left(\mathrm{mg} \cdot \mathrm{L}^{-1}\right)\end{array}$ & Detected $\left(\mathrm{mg} \cdot \mathrm{L}^{-1}\right)$ & Recovery $(\%)$ & $\begin{array}{c}\text { Relative standard } \\
\text { deviation }(\%)\end{array}$ \\
\hline 10 & $10.639 \pm 0.321$ & 106.39 & 4.597 \\
15 & $16.201 \pm 0.431$ & 108.01 & 3.510 \\
20 & $20.114 \pm 0.354$ & 100.57 & 2.376 \\
\hline
\end{tabular}

In order to decrease the effects of external factors on SERS intensity at $1272 \mathrm{~cm}^{-1}$ and take full advantage of ratio effect, SERS intensity ratio at 1272 and $1558 \mathrm{~cm}^{-1}\left(I_{1272} / I_{1558}\right)$ was used to establish the SERS calibration curve between the tetracycline concentration in duck meat extract and $I_{1272} / I_{1558}$. SERS characteristic peak at $1558 \mathrm{~cm}^{-1}$ was caused by duck meat extract. As showed from the inset in Figure 5, the linear regression equation was $y=0.0177 x+0.1213$, and the correlation coefficient $(r)$ was 0.950 , in which $x$ and $y$ represented the tetracycline concentration in duck meat extract and $I_{1272} / I_{1558}$, respectively. This illustrated that a good linearity relationship between the tetracycline concentration $(4 \sim 25 \mathrm{mg} / \mathrm{L})$ in duck meat extract and $I_{1272} / I_{1558}$ was obtained.

The SERS calibration curve obtained from above was applied to predict the tetracycline concentration in duck meat extract, and the predicted results were showed in Table 1. The average recovery of tetracycline in duck meat extract was 101 108\% with the relative standard deviation (RSD) of $2.4 \sim 4.6 \%$, which indicated that this method had a good precision and satisfactory reproducibility. The detection limit of tetracycline in duck meat extract could reach $1.120 \mathrm{mg} / \mathrm{L}$ $(S / N=3)$. The experimental results showed that the pretreatment method adopted in this paper was more simple and rapid than the conventional detection methods such as enzyme-like immunosorbent assay, microbiological method, and physical and chemical detection method. However, this method still needs to be improved owing to the disadvantage of its high detection limit, which is possibly because surface enhancement factor of SERS substrate is affected by many factors, such as particle size, shape, and uniformity of the distribution of nanoparticles [19], and the composition of duck meat can decrease intensities of tetracycline SERS characteristic peaks in duck meat extract. So, it will be a good selection, in future studies, to explore a pretreatment method with less effect on intensities of tetracycline SERS characteristic peaks in duck meat extract or a nanoparticle with the better enhancement effect to decrease the detection limit. Also, the method proposed in this paper could provide the important technical support for achieving the rapid detection of tetracycline residues in duck meat.

\section{Conclusions}

A simple and rapid method using OTR202 and OTR103 as SERS enhancement substrate was developed for the detection of tetracycline residues in duck meat. The effects of addition amount of samples and adsorption time on SERS intensity were analyzed, respectively. A good linearity relationship between the tetracycline concentration in duck meat extract 


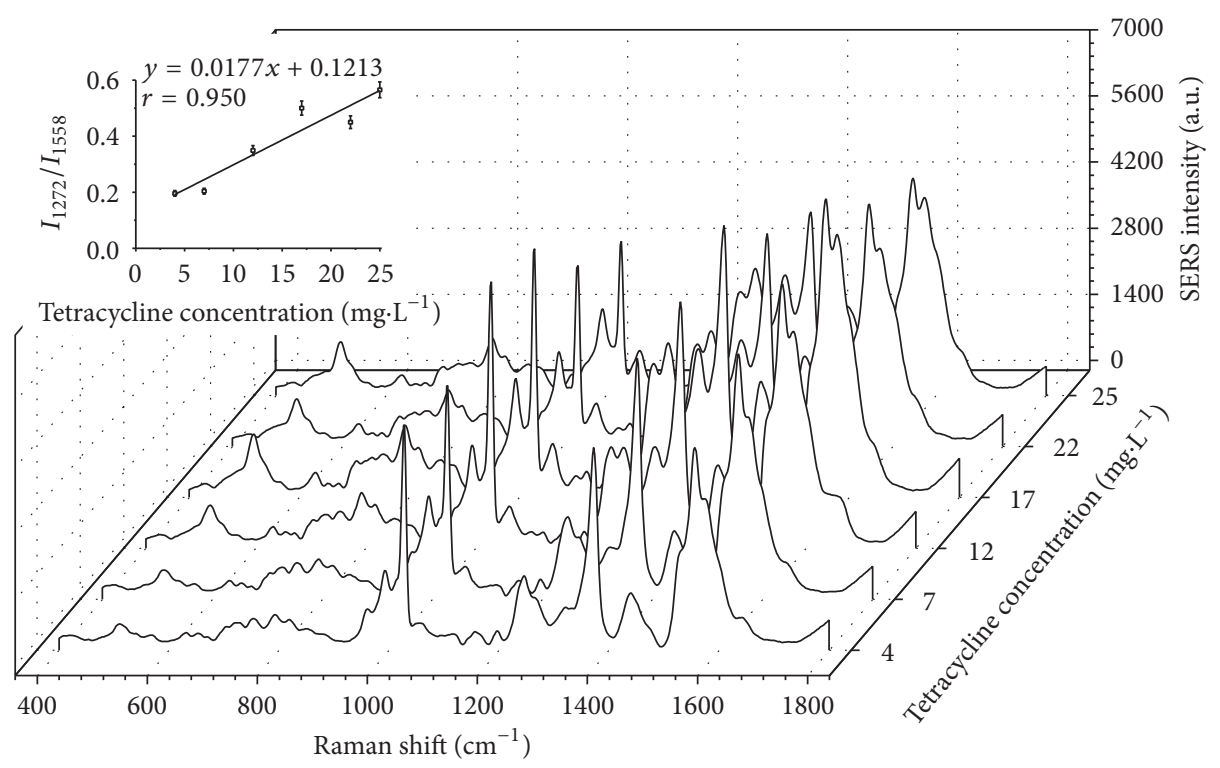

FIGURE 5: SERS spectra of duck meat extract containing different tetracycline concentrations. The inset shows the linear relationship between the tetracycline concentration and SERS intensity ratio at 1272 and $1558 \mathrm{~cm}^{-1}\left(I_{1272} / I_{1558}\right)$.

and $I_{1272} / I_{1558}$ was obtained, and the linear regression equation and $r$ were $y=0.0177 x+0.1213$ and 0.950 , respectively. The pretreatment method in this paper was simple and rapid, and the detection limit of tetracycline in duck meat extract could reach $1.120 \mathrm{mg} / \mathrm{L}$. Therefore, the method proposed in this paper was a good detection scheme for the rapid detection of tetracycline residues in duck meat.

\section{Competing Interests}

The authors declare that they have no competing interests.

\section{Authors' Contributions}

Jinhui Zhao and Ping Liu contributed equally to this work and should be considered co-first authors.

\section{Acknowledgments}

This research was supported by the National Natural Science Foundation of China (Grant no. 31660485), Science and Technology Support Project of Jiangxi Province, China (Grant no. 2012BBG70058), and External Science and Technology Cooperation Plan of Jiangxi Province, China (Grant no. 20132BDH80005).

\section{References}

[1] X. D. Gao, S. E. Chen, Y. L. Ye, X. J. Wang, J. K. Deng, and S. C. Wei, "Determination of terramycin, minocycline and aureomycin in livestock, poultry meat and salmon by high performance liquid chromatography," Journal of Food Safety and Quality, vol. 5, no. 2, pp. 369-376, 2014.

[2] J.-H. Zhao, H.-C. Yuan, M.-H. Liu, H.-B. Xiao, Q. Hong, and J. $\mathrm{Xu}$, "Rapid determination of tetracycline content in duck meat using particle swarm optimization algorithm and synchronous fluorescence spectrum," Spectroscopy and Spectral Analysis, vol. 33, no. 11, pp. 3050-3054, 2013.

[3] General Adminstration of Quality Supervision, Inspection and Quarantine of the People's of China and Standardization Administration of the People's Republic of China, 'Fresh and frozen poultry product', GB16869-2005, 2005.

[4] Y. Zhou, C.-Y. Li, Y.-S. Li et al., "Monoclonal antibody based inhibition ELISA as a new tool for the analysis of melamine in milk and pet food samples," Food Chemistry, vol. 135, no. 4, pp. 2681-2686, 2012.

[5] X. D. Yan, X. Z. Hu, H. C. Zhang, J. Liu, and J. P. Wang, "Direct determination of furaltadone metabolite, 3-amino5-morpholinomethyl-2-oxazolidinone, in meats by a simple immunoassay," Food and Agricultural Immunology, vol. 23, no. 3, pp. 203-215, 2012.

[6] J. Li, J. Liu, H.-C. Zhang, H. Li, and J.-P. Wang, "Broad specificity indirect competitive immunoassay for determination of nitrofurans in animal feeds," Analytica Chimica Acta, vol. 678, no. 1, pp. 1-6, 2010.

[7] O. G. Nagel, M. C. Beltrán, M. P. Molina, and R. L. Althaus, "Novel microbiological system for antibiotic detection in ovine milk," Small Ruminant Research, vol. 102, no. 1, pp. 26-31, 2012.

[8] P. K. Dang, G. Degand, S. Danyi et al., "Validation of a twoplate microbiological method for screening antibiotic residues in shrimp tissue," Analytica Chimica Acta, vol. 672, no. 1-2, pp. 30-39, 2010.

[9] G. F. Pang, Compilation of Official Methods of Analysis for Pesticide Residues and Veterinary Drug Residues, Standards Press of China, Beijing, China, 2009.

[10] Y. Zhang, W. Yu, L. Pei, K. Lai, B. A. Rasco, and Y. Huang, "Rapid analysis of malachite green and leucomalachite green in fish muscles with surface-enhanced resonance Raman scattering," Food Chemistry, vol. 169, pp. 80-84, 2015.

[11] Y. Y. Zhang, Y. Q. Huang, F. L. Zhai, R. Du, Y. D. Liu, and K. Q. Lai, "Analyses of enrofloxacin, furazolidone and 
malachite green in fish products with surface-enhanced Raman spectroscopy," Food Chemistry, vol. 135, no. 2, pp. 845-850, 2012.

[12] C. Li, K. Lai, Y. Zhang, L. Pei, and Y. Huang, "Use of surfaceenhanced Raman spectroscopy for the test of residuals of prohibited and restricted drugs in fish muscle," Acta Chimica Sinica, vol. 71, no. 2, pp. 221-226, 2013.

[13] H. K. Ma, X. H. Han, C. H. Zhang, X. Zhang, X. F. Shi, and J. Ma, "The study of sulfonamide antibiotics in fish based on surfaceenhanced Raman spectroscopy technology," Acta Laser Biology Sinica, vol. 23, no. 6, pp. 560-565, 2014.

[14] R. Li, H. Zhang, Q.-W. Chen, N. Yan, and H. Wang, "Improved surface-enhanced Raman scattering on micro-scale Au hollow spheres: synthesis and application in detecting tetracycline," Analyst, vol. 136, no. 12, pp. 2527-2532, 2011.

[15] C. X. Fang, J. H. Li, and Y. Z. Liang, "Determination of MTBE in gasoline by raman spectroscopy combined with baseline correction method," Journal of Instrumental Analysis, vol. 31, no. 5, pp. 541-545, 2011.

[16] X. Li and Y. Lü, "Background subtraction in raman measurement of ethanol concentration," Journal of Beijing Information Science and Technology University, vol. 28, no. 2, pp. 27-30, 2013.

[17] X. Y. Chen, Study on Antibiotics Raman Spectroscopy Based on Partial Least Squares, Tianjin University, Tianjin, China, 2012.

[18] C. F. Leypold, M. Reiher, G. Brehm et al., "Tetracycline and derivatives-assignment of IR and Raman spectra via DFT calculations," Physical Chemistry Chemical Physics, vol. 5, no. 6, pp. 1149-1157, 2003.

[19] F.-L. Zhai, Y.-Q. Huang, X.-C. Wang, and K.-Q. Lai, "Surfaceenhanced Raman spectroscopy for rapid determination of $\beta$ agonists in swine urine," Chinese Journal of Analytical Chemistry, vol. 40, no. 5, pp. 718-723, 2012. 

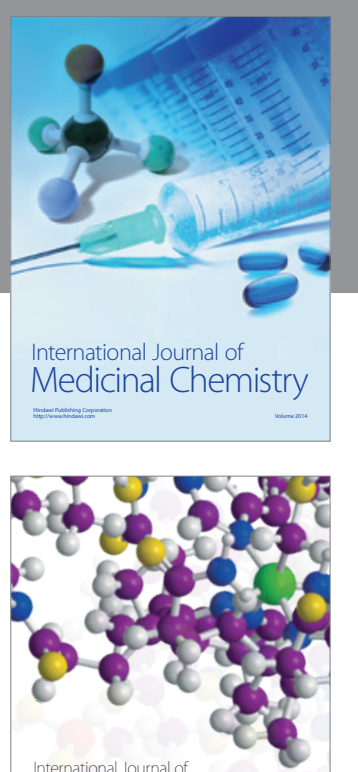

Carbohydrate Chemistry

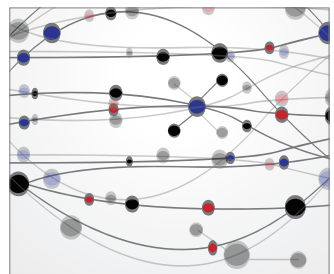

The Scientific World Journal
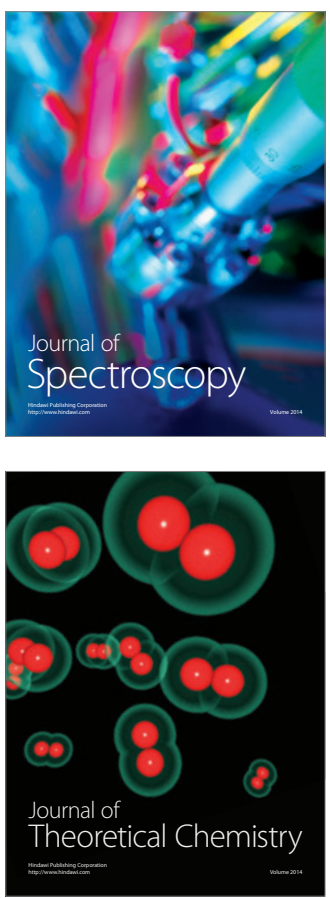
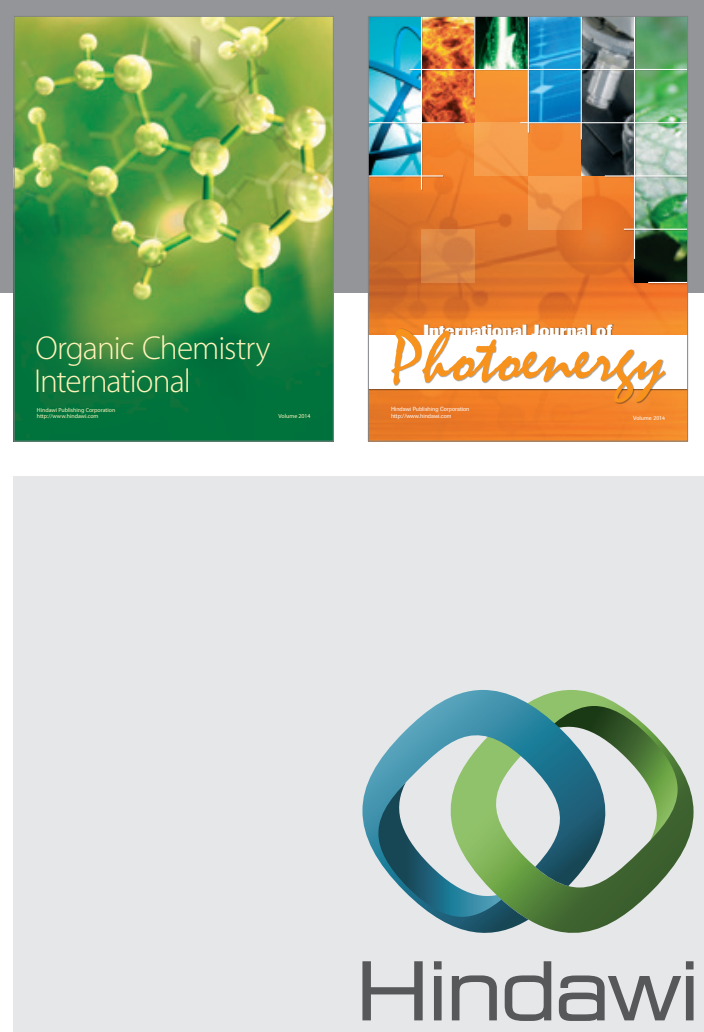

Submit your manuscripts at

http://www.hindawi.com

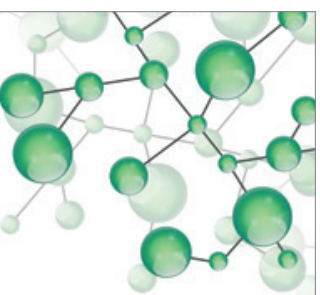

International Journal of

Inorganic Chemistry

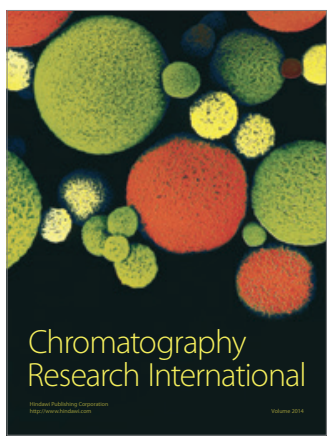

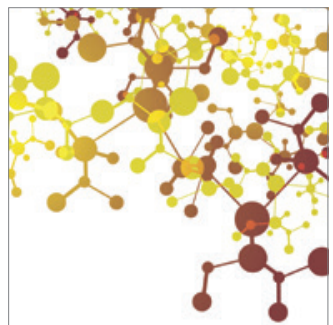

Applied Chemistry
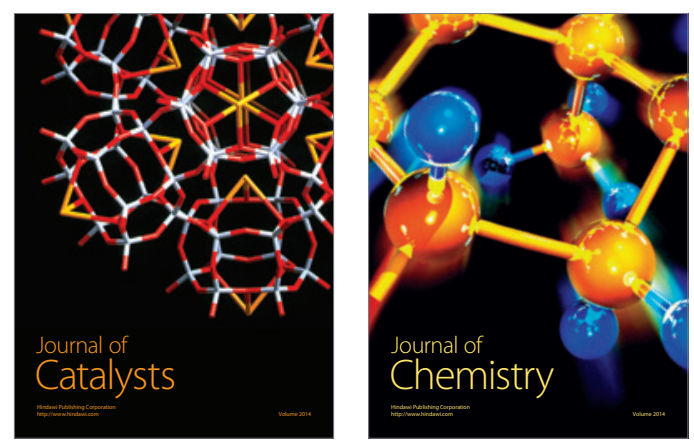
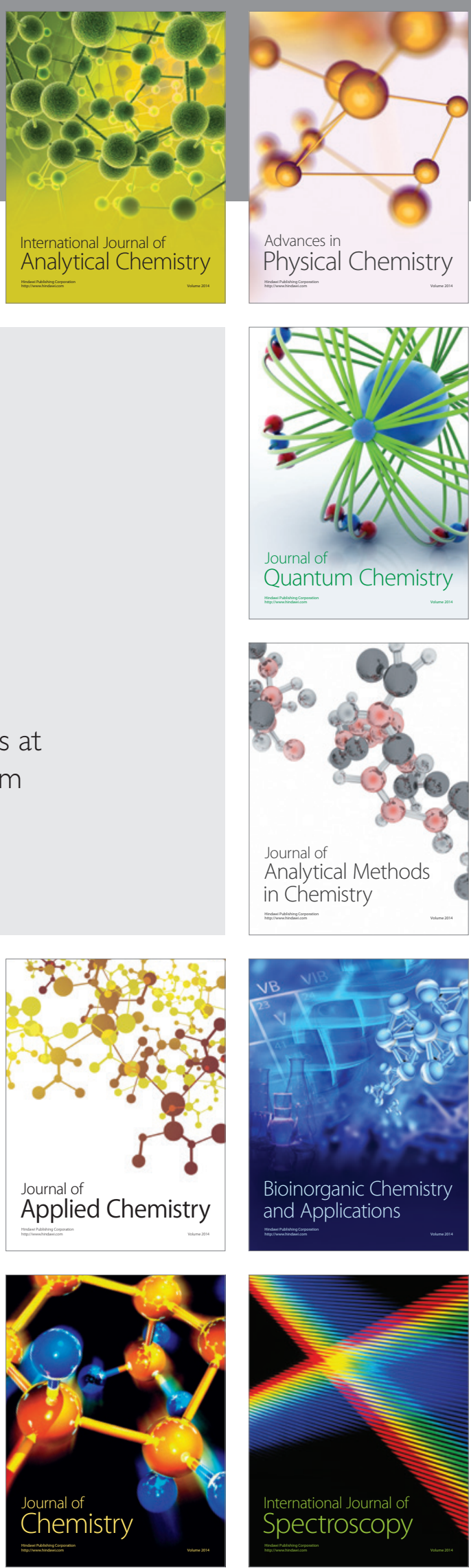